\title{
ON THE GROWTH OF SOLUTIONS IN THE OSCILLATORY CASE
}

\section{ROBERT M. KAUF FMAN}

ABSTRACT. Suppose that $A$ is a bounded continuously differentiable function from $[0, \infty)$ to the real $n \times n$ Hermitian matrices such that, for every $\epsilon>0$ and every $\lambda>0$, there is an $a$ (depending on $\epsilon$ and $\lambda$ ) such that $D^{2}-A-\epsilon E$ and $D^{2}+A^{\prime} / \lambda-\epsilon E$ are disconjugate on $[a, \infty)$, where $E$ is the $n \times n$ identity matrix. It follows from the result of this paper that no solution of $\left(D^{2}+A\right) f=0$ can either grow or decay exponentially.

A simplified version of the result of this paper is as follows: Suppose $A$ is a continuously differentiable, bounded function from $[0, \infty)$ to the positive semidefinite Hermitian complex matrices. Suppose $A^{\prime}(t)$ converges to the zero matrix as $t$ approaches infinity. Then no solution of the equation $\left(D^{2}+A\right) f=0$ either grows or decays exponentially, where $D=d / d t$. The complete version is some what more general, in that the conditions that $A(t)$ be positive semidefinite for every $t$ and that $A^{\prime}(t)$ converge to zero as $t$ approaches infinity are weakened somewhat. A corollary of this result is that, when it applies, the essential spectrum of any operator in $L_{2}[0, \infty)$ associated with $D^{2}+A$ contains $(-\infty, 0]$. Both the result and its corollary appear to be new even in the scalar case. They are perhaps of interest in view of the wellknown example (see Bellman [1, p. 113]) which shows that even under much stronger hypotheses, it need not be true that all solutions are bounded, even in the scalar case.

It should perhaps be remarked that proving the result in the general case, rather than just the scalar case, is worthwhile because it permits a much wider range of possible applications to mechanics.

Notation. A function $f$ from $[a, \infty)$ into complex $n$ dimensional space $C^{n}$ is said to be in $C_{0}^{\infty}(a, \infty)$ if it is infinitely differentiable and supported in a compact subset of $(a, \infty)$.

Notation. $L_{2}^{n}[0, \infty)$ denotes the space of measurable functions $f$ from

Received by the editors May 9, 1974.

AMS (MOS) subject classifications (1970). Primary 34C10, 34B25.

Key words and phrases. Exponential growth, Fredholm operator, disconjugacy, essential spectrum. 
$[0, \infty)$ into complex $n$ dimensional space $C^{n}$ such that $\langle f, f\rangle$ is in $L_{1}[0, \infty)$, where $\langle$,$\rangle denotes the dot product in C^{n} . L_{2}^{n}[0, \infty)$ is clearly a Hilbert space, with inner product denoted by $($,$) .$

Notation. Throughout the paper, let $S$ denote the set of all $f$ in $L_{2}^{n}[0, \infty)$ such that $D f$ is absolutely continuous and $D^{2} f$ is in $L_{2}^{n}[0, \infty)$, where $D=d / d t$. It is well known that, for $f \in S, D f \in L_{2}^{n}[0, \infty)$.

Definition. Let $L=D^{2}+\lambda D+B$, where $\lambda$ is a real number, and $B$ be a bounded continuous function from $[0, \infty)$ to the $n \times n$ complex Hermitian matrices. Let $L_{M}$ denote the restriction of $L$ to $S, L_{M}$ is called the maximal operator associated with $L$.

Notation. Let $L$ be as in the previous definition. Then $L^{+}$denotes the expression $D^{2}-\lambda D+B$.

Notation. Let $S_{0}$ denote the set of all $f$ in $S$ such that $f(0)=D f(0)=0$.

Definition. Let $L$ be as in the above definitions. Let $L_{0}$ denote the restriction of $L$ to $S_{0} . L_{0}$ is called the minimal operator associated with $L$.

Theorem 1. Suppose that $L=D^{2}+\lambda D+B$, where $\lambda$ is real and $B$ is a continuous, bounded function from $[0, \infty)$ to the complex $n \times n$ Hermitian matrices. Then $\left(L_{0}\right)^{*}=\left(L^{+}\right)_{M}$.

Notation. Let $L_{R}$ denote the restriction of $L$ to $C_{0}^{\infty}(0, \infty)$.

Theorem 2. Let $L$ be as in Theorem 1. Then $L_{0}$ is the closure of $L_{R}$.

Remark. Both of the preceding theorems are well known in the scalar case, and seem to be fairly well known in the present context, even though the proofs do not seem to have appeared in print except in the scalar case. The general case can be proved fairly easily by using the scalar case, or alternatively by imitating the methods of Dunford and Schwartz [3] in the scalar case.

Theorem 3. Suppose that range $L_{0}$ is closed, where $L$ is as in Theorems 1 and 2. Then nullity $L_{M}+$ nullity $\left(L^{+}\right)_{M}=2 n$.

Proof. Clearly $L_{M}$ is a $2 n$ dimensional extension of $L_{0}$, so range $L_{M}$ is closed. It follows from Goldberg [4, Theorem IV. 1.2, p. 95] that range $\left(L^{+}\right)_{0}$ and range $\left(L^{+}\right)_{M}$ are both closed. The index of a closed operator is defined to be its nullity minus the deficiency of its range. (See Goldberg [4, $\{I V .2]$ for a discussion of the index.) It follows that index $L_{M}=$ index $L_{0}+2 n$, since a one dimensional extension raises the index by 1 . But the index of $\left(L_{0}\right)^{*}$ is - index $L_{0}$. Also, since $L_{0}$ has no nontrivial 
null space, $\left(L^{+}\right)_{M}$ is surjective. Thus index $L_{0}=-$ nullity $\left(L^{+}\right)_{M}$. Furthermore, index $L_{M}=$ nullity $L_{M}$. The conclusion follows.

Definition. A Fredholm operator is a closed linear operator with closed range, finite nullity, and finite deficiency. The index of a Fredholm operator is its nullity minus the deficiency of its range.

Theorem 4. Suppose $T$ is a Fredholm operator. Suppose that domain B contains domain $T$, where $B$ is a closed linear operator. Then there is a $\delta>0$ such that, if $|\lambda|+|\alpha|<\delta$, index $T+\lambda B+\alpha I=$ index $T$, where $I$ is the identity operator.

Theorem 4 is a special case of [4, Theorem V.3.6, p. 122] since, by Theorem V.3.3, $B$ is $T$ bounded.

Notation. Throughout this paper, $I$ denotes the identity operator in $L_{2}^{n}[0, \infty)$ and $E$ denotes the $n \times n$ identity matrix.

Definition. Let $A$ be a continuous function from $[0, \infty)$ to the complex $N \times N$ Hermitian matrices. $D^{2}+A$ is said to be nonpositive on $[a, \infty)$ if $\left(\left(D^{2}+A\right) f, f\right) \leq 0$ for all $f$ which are in $C_{0}^{\infty}$.

Remark. If $A(t)$ is a real Hermitian matrix for each $t$, nonpositivity is equivalent to disconjugacy, as is shown in Coppel [3, Theorem 14, p. 61] plus a simple density argument to pass from $C_{0}^{\infty}(a, b)$ to the absolutely continuous $f$ vanishing at the end points of $[a, b]$. The oscillation theory of second order selfadjoint systems, and even more general systems, is discussed in detail in Chapter 2 of Coppel. Our main result can be thought of, loosely, as relating the growth of solutions of $\left(D^{2}+A\right) f=0$ to the oscillation theory of $\left(D^{2}+A\right) f=0$ and $\left(D^{2}+A^{\prime}\right) f=0$.

Definition. Let $B$ be a continuous function from $[0, \infty)$ to the $n \times n$ complex Hermitian matrices. $B$ is said to have property $\mathrm{P}$ if there is some real number $\lambda<1$ such that, for every $\epsilon>0$, there is a $b$ (which depends upon $\epsilon$ ) such that both $D^{2}+B-\epsilon E$ and $D^{2}+B / \lambda-\epsilon E$ are nonpositive on $[b, \infty)$.

Remark. If $B(t)$ converges to zero as $t$ approaches infinity, then $B$ has property $\mathrm{P}$.

We now come to our ma in result.

Theorem 5. Suppose that $A$ is a continuously differentiable bounded function from $[0, \infty)$ to the complex $n \times n$ Hermitian matrices. Suppose the set of real numbers $\lambda$ such that $A^{\prime} / \lambda$ has property $\mathrm{P}$ contains $(-\infty, 0)$ or $(0, \infty)$. Suppose further that, for every $\epsilon>0$, there is a positive number a 
(which depends upon $\epsilon$ ) such that $D^{2}-A-\epsilon E$ is nonpositive on $[a, \infty)$. Then, for every $\lambda>0$, and for every solution $f$ of the equation $\left(D^{2}+A\right) f=0$, $e^{-\lambda t} f(t)$ converges to zero as $t$ approaches infinity, and $e^{-\lambda t} f^{\prime}(t)$ converges to zero as $t$ approaches infinity, but $e^{\lambda t} f(t)$ is not in $L_{2}[0, \infty)$.

Proof. We need several lemmas.

Lemma 1. Suppose that $B$ has property P. Suppose $f_{n}$ is a sequence of functions in $C_{0}^{\infty}(n, \infty)$ with $\left\|f_{n}\right\|=1$, and such that, for every $\epsilon>0$, $\left(\left(D^{2}+B\right) f_{n}, f_{n}\right)$ becomes greater than $-\epsilon$ as $n$ becomes large. Then $\left(\left(D^{2}+B\right) f_{n}, f_{n}\right)$ converges to zero, and also $\left\|D f_{n}\right\|$ converges to zero.

Proof. First, it is clear that $\left(\left(D^{2}+B\right) f_{n}, f_{n}\right)$ converges to zero, since, for each $\epsilon>0$, we have $\left(\left(D^{2}+B-\epsilon E\right) f_{n}, f_{n}\right)$ becoming eventually less than zero as $n$ becomes large, since $B$ has property $\mathrm{P}$. Suppose that $\left(D f_{n}, D f_{n}\right)$ does not converge to zero. Then $\left(B f_{n}, f_{n}\right)$ is eventually $\geq \delta$, for some $\delta>0$, since $\left(\left(D^{2}+B\right) f_{n}, f_{n}\right)$ converges to zero. Now

$$
\left(\left(D^{2}+B / \lambda\right) f_{n^{\prime}} f_{n}\right)=\left(\left(D^{2}+B\right) f_{n^{\prime}} f_{n}\right)+(1 / \lambda-1)\left(B f_{n^{\prime}} f_{n}\right),
$$

and thus $\left(\left(D^{2}+B / \lambda\right) f_{n}, f_{n}\right)$ is eventually $\geq((1 / \lambda) \delta-\delta) / 2$. This contradicts property $P$.

Lemma 2. Suppose that $B$ is a continuously differentiable function from $[0, \infty)$ to the Hermitian complex matrices such that eit her $B^{\prime} / \lambda$ or $-B^{\prime} / \lambda$ has property $\mathrm{P}$. Suppose also that, for some $\epsilon>0$, there is an a such that $D^{2}-B+\epsilon E$ is nonpositive on $[a, \infty)$. Then $\left(D^{2}+\lambda D+B\right)_{0}$ has closed range.

Proof. Let $Q=D^{2}+\lambda D+B$. Then

$$
Q^{+} Q=\left(D^{2}+B\right)^{2}-\lambda^{2} D^{2}-\lambda B^{\prime}=\left(D^{2}+B\right)^{2}-\lambda^{2}\left(D^{2}+B^{\prime} / \lambda\right) \text {. }
$$

Suppose that $B^{\prime} / \lambda$ has property P. Suppose also that range $Q_{0}$ is not closed. Then there is a sequence $f_{n}$ in $C_{0}^{\infty}(n, \infty)$ with $\left\|f_{n}\right\|=1$ and $\left\|Q f_{n}\right\|$ converging to zero. (The fact that the $f_{n}$ can be chosen with compact support in $(n, \infty)$ follows from the fact that if, on any interval $[n, \infty), R_{0}$ had closed range, where $R$ is the restriction of $Q$ to $[n, \infty)$, then $Q_{0}$ would have closed range.)

Since $\left(Q^{+} Q f_{n}, f_{n}\right)$ converges to zero, and since $\left(\left(D^{2}+B\right)^{2} f_{n}, f_{n}\right) \geq 0$, it follows from our formula for $Q^{+} Q$ that, for any $\epsilon>0,\left(\left(D^{2}+B^{\prime} / \lambda\right) f_{n}, f_{n}\right)$ eventually becomes larger than $-\epsilon$. By Lemma 1 , both $\left(D^{2} f_{n}, f_{n}\right)$ and 
$\left(\left(D^{2}+B^{\prime} / \lambda\right) f_{n}, f_{n}\right)$ converge to zero. Once again using the formula for $Q^{+} Q$, we see that $\left\|\left(D^{2}+B\right) f_{n}\right\|$ converges to zero. But then $\left(B f_{n}, f_{n}\right)$ also converges to zero. Thus, for any $\epsilon>0,\left(\left(D^{2}-B+\epsilon E\right) f_{n}, f_{n}\right)$ is eventually positive, which contradicts the hypotheses of the lemma. Thus range $Q_{0}$ is closed, and Lemma 2 is proved, provided that $B^{\prime} / \lambda$ has property $P$. If $-B^{\prime} / \lambda$ has property $P$, we use what we have proved to show that range $\left(Q^{+}\right)_{0}$ is closed, since $Q^{+}=D^{2}-\lambda D+B$. But, if range $\left(Q^{+}\right)_{0}$ is closed, then range $\left(Q^{+}\right)_{M}$ is closed, since a finite dimensional extension of a closed subspace is closed. Then, since it is well known (see [4, IV.1.8, p. 98]) that the adjoint of a closed operator with closed range also has closed range, it follows from the fact that $\left(\left(Q^{+}\right)_{M}\right)^{*}=\left(\left(Q_{0}\right)^{*}\right)^{*}=Q_{0}$ that range $Q_{0}$ is closed.

Proof of Theorem 5. For any nonzero real number $\lambda$, define the expression $Q_{\lambda}$ by $Q_{\lambda} f(t)=e^{-\lambda t} L\left(e^{\lambda t} f(t)\right)$, where $L$ denotes $D^{2}+A$. When expanded, $Q_{\lambda}=D^{2}+2 \lambda D+A+\lambda^{2} E$. Let $\epsilon=\lambda^{2} / 2$. By hypothesis, $D^{2}-A-\epsilon E$ is nonpositive on some interval $(a, \infty)$. If $B=A+\lambda^{2} E$, then $D^{2}-A-\epsilon E=$ $D^{2}-B+\epsilon E$, so $B$ satisfies the hypotheses of Lemma 2 .

Therefore, range $\left(Q_{\lambda}\right)_{0}$ is closed for any $\lambda \neq 0$, and thus each $\left(Q_{\lambda}\right)_{M}$ is surjective (since its adjoint, $\left(\left(Q_{\lambda}\right)^{+}\right)_{0}$, has no nontrivial null space). Each $\left(Q_{\lambda}\right)_{M}$ is a Fredholm operator, with domain $S$ (see notation at the beginning of the paper). It follows from Theorem 4 that index $\left(Q_{\lambda}\right)_{M}$ is constant for every $\lambda>0$, and also for every $\lambda<0$, though the se two constants need not be the same. But index $\left(Q_{\lambda}\right)_{M}$ is the dimension of the $L_{2}^{n}[0, \infty)$ solution space of the equation $Q_{\lambda} g=0$. Also, if $Q_{\lambda} g=0$, then $g(t)=e^{-\lambda t} f(t)$, where $\left(D^{2}+A\right) f=0$. If $\lambda$ is large enough, then any such $g$ is in $L_{2}^{n}[0, \infty)$, since $A^{*}$ is bounded. Thus, for all $\lambda>0$, nullity $\left(Q_{\lambda}\right)_{M}=2 n$. Therefore, by Theorem 3, for $\lambda<0$ it follows that nullity $\left(Q_{\lambda}\right)_{M}=0$, since $\left(Q_{\lambda}\right)^{+}=Q_{-\lambda}$. We have shown that for any solution $f$ of the equation $\left(D^{2}+A\right) f=0$, $e^{-\lambda t} f(t)$ is in $L_{2}^{n}[0, \infty)$, but $e^{\lambda t} f(t)$ is not in $L_{2}^{n}[0, \infty)$. To complete the proof, we need only to show that, as $t$ approaches infinity, $e^{-\lambda t} f(t)$ converges to zero.

We have shown that $e^{-\lambda t} f(t) \in S$. Therefore $D\left(e^{-\lambda t} f(t)\right)$ is in $L_{2}^{n}[0, \infty)$. However, if $h$ is any complex valued, absolutely continuous function, and $h$ and $h^{\prime}$ are both in $L_{2}[a, \infty), h(a)=\delta,\left\|h^{\prime}\right\|=M$, it follows that $h(x) \geq \delta / 2$ for all $x \in[a, a+\delta / 2 M]$, so that $\|h\| \geq \delta^{2} / 4 M$. If $h$ is in $L_{2}[0, \infty)$, it follows that, as a gets large, $h(a)$ converges to zero. This shows that $e^{-\lambda t} f(t)$ converges to zero as $t$ approaches infinity. But since $D\left(e^{-\lambda t} f(t)\right)=$ $-\lambda e^{-\lambda t} f(t)+e^{-\lambda t} f^{\prime}(t)$, and since 


$$
\begin{aligned}
D^{2}\left(e^{-\lambda t} f(t)\right) & =-2 \lambda e^{-\lambda t} f^{\prime}(t)+e^{-\lambda t} f^{\prime \prime}(t)+\lambda^{2} e^{-\lambda t} f(t) \\
& =-2 \lambda e^{-\lambda t} f^{\prime}(t)+e^{-\lambda t} A(t) f(t)+\lambda^{2} e^{-\lambda t} f(t),
\end{aligned}
$$

we see that $e^{-\lambda t} f^{\prime}(t)$ is in $L_{2}^{n}[0, \infty)$, because $D\left(e^{-\lambda t} f(t)\right)$ is, and therefore $D^{2}\left(e^{-\lambda t} f(t)\right)$ is in $L_{2}^{n}[0, \infty)$, so that $D\left(e^{-\lambda t} f(t)\right)$ converges to zero as $t$ approaches infinity, and thus $e^{-\lambda t} f^{\prime}(t)$ converges to zero as $t$ approaches infinity. Theorem 5 is proved.

Corollary. Let $A$ be as in Theorem 5. Let $H$ be any selfadjoint extension of $\left(D^{2}+A\right)_{0}$. Then the essential spectrum of $H$ contains the interval $(-\infty, 0]$.

Proof. If the range of $\left(D^{2}+A\right)_{M}$ were closed, Theorem 4 would imply that nullity $\left(Q_{\lambda}\right)_{M}$ would be constant in a neighborhood of $\lambda=0$. But this would imply that, for small $\lambda$, nullity $\left(Q_{-\lambda}\right)_{M}=$ nullity $\left(Q_{\lambda}\right)_{M}$, or in other words that $0=2 n$.

Therefore, for any $A$ satisfying the hypotheses of Theorem 5, range $\left(D^{2}+A\right)_{M}$ is not closed. But, if $A$ satisfies the hypotheses of Theorem 5, then so does $A+\lambda E$ for any $\lambda>0$. Furthermore, since $\left(D^{2}+A+\lambda E\right)_{M}$ is a finite dimensional extension of $H+\lambda I$, if the range of $H+\lambda I$ were closed, then range $\left(D^{2}+A+\lambda E\right)_{M}$ would be closed. Since the essential spectrum of $H$ is the set of all $\lambda$ such that $H-\lambda I$ does not have closed range, the conclusion follows.

\section{REFERENCES}

1. R. Bellman, Stability theory of differential equations, McGraw-Hill, New York, 1953. MR 15, 794.

2. W. A. Coppel, Disconjugacy, Lecture Notes in Math., vol. 220, SpringerVerlag, New York, 1971.

3. N. Dunford and J. T. Schwartz, Linear operators. II: Spectral theory. Selfadjoint operators in Hilbert space, Wiley, New York, 1963. MR $32 \sharp 6181$.

4. S. Goldberg, Unbounded linear operators: Theory and applications, McGrawHill, New York, 1966. MR 34 \#580.

DEPARTMENT OF MATHEMATICS AND COMPUTER SCIENCE, WESTERN WASHINGTON STATE COLLEGE, BELLINGHAM, WASHINGTON 98225 